\title{
Viability and Functionality of Primordial Germ Cells after Freeze-thaw in Chickens
}

\author{
Yoshiaki Nakamura ${ }^{1,2,3}$, Fumitake Usui ${ }^{1}$, Daichi Miyahara ${ }^{1}$, Takafumi Mori ${ }^{1}$, Haruhi Watanabe ${ }^{1}$, \\ Tamao Ono ${ }^{1}$, Kumiko Takeda ${ }^{2}$, Keijiro Nirasawa ${ }^{2}$, Hiroshi Kagami ${ }^{1}$ and Takahiro Tagami ${ }^{2}$ \\ ${ }^{1}$ Faculty of Agriculture, Shinshu University, Minamiminowa, Nagano 399-4598, Japan \\ ${ }^{2}$ National Institute of Livestock and Grassland Science, Tsukuba, Ibaraki 305-0901, Japan \\ ${ }^{3}$ Research Fellow of the Japan Society for the Promotion of Science, Japan
}

\begin{abstract}
The purpose of this study is to clarify the effects of freeze-thaw treatment on viability and functionality of primordial germ cells (PGCs) in chickens. PGCs were collected separately from embryonic blood of White Leghorn, Barred Plymouth Rock and Fayoumi breeds. Some PGCs were labeled with the fluorescent lipophilic carbocyanine dye to analyze functionality by transfer assay. $100 \mathrm{PGCs}$ were used for each experiment to ensure accuracy of the test results. In the experimental group, PGCs were slow-frozen, then stored in liquid nitrogen for 1 month. In the unfrozen control group, PGCs were utilized immediately. The recovery rate of PGCs after freeze-thaw was 54.3\%. The viability of PGCs in the frozen group was significantly lower than that of the control group $(P<0.05)(85.7 \%$ vs. $99.2 \%)$ with no significant difference between the three breeds. Thirty fluorescent-labeled PGCs were randomly chosen from each set of recovered PGCs after freeze-thaw in the frozen group, and from each set of 100 PGCs in the control group, for transfer into the bloodstream. Gonadal migration of transferred PGCs was observed in all embryos in both test and control groups. The number of PGCs settled in the gonads of embryos at stage 27 was $52.8 \%$ lower in the frozen group than in the unfrozen control group $(P<0.05)$. In the control group, significant difference in establishment of PGCs in stage 27 gonads was observed between the three breeds, with White Leghorn chickens harboring the most PGCs, and Barred Plymouth Rock chickens the fewest. We conclude that freeze-thaw treatment causes a decrease in functionality of PGCs in chicken, with only an estimated $46.5 \%$ of frozen PGCs being viable after thawing.
\end{abstract}

Key words: chickens, cryopreservation, functionality, primordial germ cells, viability

J. Poult. Sci., 48: 57-63, 2011

\section{Introduction}

The collection and preservation of genetic materials obtained from existing animal species is urgently needed, since genetic materials are unrecoverable once the animals have become extinct. At present, livestock are costly to feed and livestock populations are vulnerable to disease outbreaks and environmental disasters. Cryopreservation of cells and/or tissues obtained from remaining animals is regarded as an available means of overcoming these problems. Recent advances in freezing techniques enable cells to be conserved as genetic resources semi-permanently, without changing their genetic characteristics. However, to disseminate these genetic materials at the cellular level, techniques for regenerating live animals obtained from conserved cells must be developed. In chickens, the cryo-

Received: September 6, 2010, Accepted: October 5, 2010

Released Online Advance Publication: November 25, 2010

Correspondence: Dr. Takahiro Tagami, National Institute of Livestock and Grassland Science, 2 Ikenodai, Tsukuba, Ibaraki 305-0901, Japan. (E-mail: tagami@affrc.go.jp) preservation of semen has been achieved (Lake and Stewart, 1978; Hammerstedt and Graham, 1992), but ova are not yet available for freezing in the same way because of their large size and their yolk-laden structure. Therefore, cryopreservation of male gametes allows the recovery of only single genes from chickens at the present time.

The first identifiable precursor cells for gametes are primordial germ cells (PGCs). In chickens, approximately $30-150$ PGCs are found in the central zone of area pellucida of stage $X$ embryos, which contain about 3-6 $\times$ $10^{4}$ cells (Tsunekawa et al., 2000; Nakamura et al., 2007; Eyal-Giladi and Kochav, 1976). Following formation of the primitive streak, PGCs migrate to the anterior edge of the extraembryonic region, the so-called germinal crescent region. As blood vessels develop in the germinal crescent region, PGCs enter the vascular network and are transported by the embryonic circulation to the future gonadal region (Nakamura et al., 2007). Recently, PGCs have gained much attention as an available genetic resource in chickens. Germline chimeric chickens have been produced by transferring donor PGCs from isolated sites to 
the same or different locations in recipient embryos, where they differentiated normally into functional gametes (Petitte et al., 1990; Tajima et al., 1993; Vick et al., 1993; Kagami et al., 1997; Park et al., 2003). Several techniques for purifying PGCs obtained from embryos have been established: Ficoll density gradient centrifugation (Yasuda et al., 1992), immunomagnetic cell sorting (Ono and Machida, 1999), fluorescence-activated cell sorting (Mozdziak et al., 2005), ammonium chloride-potassium lysis process (Yamamoto et al., 2007) and Nycodenz density gradient centrifugation (in mouse: Mayanagi et al., 2003, in chicken: Zhao and Kuwana, 2003). It has been reported that frozen-thawed PGCs give rise to functional gametes via germline chimeric chickens (Naito et al., 1994a; Tajima et al., 1998; Kuwana et al., 2006; Nakamura et al., 2010a). These studies support the idea that cryopreservation of PGCs in liquid nitrogen can preserve both female and male chicken genetic material for a relatively long time.

To create better conditions for enhancing PGC-mediated genetic resource conservation systems in chickens, the utility of frozen-thawed PGCs must be examined. The aim of the present study, therefore, is to clarify the effects of freezing and thawing on viability and functionality of chicken PGCs. The results obtained from this study will yield important information regarding the conservation of PGC-based avian genetic resources.

\section{Materials and Methods}

\section{Fertilized Eggs and Animal Care}

Fertilized eggs obtained from White Leghorn (WL), Barred Plymouth Rock (BPR) and Fayoumi (FA) chickens, maintained at the National Institute of Livestock and Grassland Science (NILGS), were reproduced by artificial insemination. All animal care and use in this study was conducted in accordance with the animal experimentation guidelines of our institute (NILGS Animal Care Committee).

\section{Collection of Embryonic Blood and Harvest of PGCs}

Freshly obtained fertilized eggs were incubated at $39.0^{\circ} \mathrm{C}$ and relative humidity 50 to $60 \%$, with tilting by $90^{\circ}$ twice an hour, in a forced air incubator (P-008B Biotype; Showa Furanki, Saitama, Japan) for 50 to $54 \mathrm{~h}$ to obtain embryos at stages 14 to 16 (Hamburger and Hamilton, 1951). The blood of the whole embryo was collected from the dorsal aorta and terminal vein using a fine glass micropipette under a microscope (MS5; Leica Microsystems). The collected blood was pooled in phosphate buffered saline without $\mathrm{Ca}^{2+}$ and $\mathrm{Mg}^{2+}$ (PBS (-)) separately for each chicken breed. PGCs were then concentrated by Nycodenz density gradient centrifugation (Zhao and Kuwana, 2003), with minor modifications; namely, 10\% fetal bovine serum (FBS) dissolved in PBS $(-)(10 \%$ FBS in PBS (-)) replaced the standard KAv1 medium (Kuwana et al., 1996) as a buffer. To assay the migration capability of PGCs to the gonads, some concentrated cells were labeled with the fluorescent lipophilic carbocyanine dye PKH-26 (Zynaxis, Malvern, PA) ac- cording to the protocol of Yamamoto et al. (2007). The chicken PGCs were distinguishable from blood cells by their large size and by the presence of numerous refractive granules in their cytoplasm, as observed under phase contrast microscopy (Fujimoto et al., 1976). Morphologically normal PGCs were collected through a fine glass micropipette from the concentrated cell suspension under the phase contrast microscope (IX71, OLYMPUS, Tokyo, Japan). Sets of 100 PGCs were randomly divided into the frozen test group and the unfrozen control group.

\section{Freezing and Thawing of PGCs}

In the frozen group, PGCs were separately suspended in $200 \mu$ L of Cell Banker 1 (Juji Field, Tokyo, Japan) in a 1.2 $\mathrm{m} l$ cryotube (Nalgene, NY, USA), and then readily frozen. In the unfrozen control group, PGCs were used immediately for viability test or transplantation. The cryotubes were frozen using a freezing container (Nalgene, NY, USA) in a deep freezer at $-80^{\circ} \mathrm{C}$ overnight. Thereafter, the cryotubes were stored in liquid nitrogen $(-196$ ${ }^{\circ} \mathrm{C}$ ) for 1 month. After storage, the cryotubes were removed from the liquid nitrogen and immediately placed in water at $39^{\circ} \mathrm{C}$ until the ice had melted. After thawing, the cell suspension was diluted in $1 \mathrm{ml}$ of $10 \%$ FBS in PBS (-) and centrifuged at $200 \mathrm{~g}$ for $4 \mathrm{~min}$ to remove the cryoprotectant. The supernatant was discarded and approximately $20 \mu l$ of cell suspension was placed in a plastic dish. The number of recovered PGCs after freeze-thaw was counted under a microscope (Eclipse E1000, Nikon, Tokyo, Japan), and expressed as recovery rate. After counting, the cells were utilized for viability test or transfer.

\section{Viability Test of PGCS}

Viability assays were conducted on both the frozen group ( $\sim 20 \mu l$ cell suspension), and on the control group ( $\sim 20 \mu l$ cell suspensions of 100 PGCs in modified buffer). $0.4 \%$ Trypan blue solution $(10 \mu l)$ was then added to each drop of PGC suspension and the mixture incubated for 2 min at room temperature. The viability of the PGCs in both groups was determined by the Trypan blue exclusion method (Freshney, 1987).

\section{Transfer of PGCs}

WL embryos were prepared as recipients and then incubated for 48 to $52 \mathrm{~h}$ until they reached stage 14 under the conditions described above. 30 PGCs labeled with PKH-26 were randomly chosen from each set of recovered PGCs after freeze-thaw in the frozen group, and from each set of 100 PGCs in the control group. These sets of 30 PGCs were microinjected through the dorsal aorta into the bloodstream of WL embryos. Manipulated embryos were incubated for 5 days (up to stage 27), after which whole gonads were collected. The number of PGCs labeled with PKH-26 settled in the gonads was counted under fluorescence microscopy (DFC480-Note OY, Leica Microsystems).

Statistical Analysis

Data were presented as the least squares mean (LSM) \pm SEM. Differences in viability and in number of PGCs 
labeled with PKH-26 were compared between frozen and control groups using two-way ANOVA. Recovery rate, viability and the number of PGCs labeled with PKH-26 between each of the three breeds was also analyzed by two-way ANOVA. If model effect was found to be significant, differences between mean values for each treatment were evaluated using Bonferroni's test. Significance was set at $P<0.05$.

\section{Results}

\section{Recovery Rate and Viability of PGCs after Freezing and Thawing}

The number of recovered and viable PGCs after freezing and thawing is shown in Table 1. No significant differences in the number of recovered and viable PGCs after freeze-thaw was observed between the three breeds. The average rate of viable PGCs, as measured by the Trypan blue exclusion method, was significantly lower for the frozen group than for the unfrozen control group (85.7\% versus $99.2 \% ; P<0.05$ ) (Table 2 ).

\section{Migration Capability of PGCs to the Gonads after Freezing and Thawing}

To analyze the functionality of PGCs after freeze-thaw treatment, sets of 30 fluorescent-labeled cells were transferred into the bloodstreams of recipient WL chicken embryos. Gonadal migration of transferred PGCs from both frozen and control groups was observed in all manipulated embryos. However, the number of PGCs in stage 27 gonads transferred from the frozen group was significantly lower than the control group, regardless of breed $(P<0.05)$ (Figure; Table 3$)$. In the unfrozen group, moreover, the number of PGCs settled in stage 27 gonads was dependent on breed $(P<0.05)$, with average PGC count highest for WL embryos, and lowest for BPR embryos (Table 3). Although the same trend was observed in the frozen group, the differences were not statistically significant.

\section{Discussion}

It has been reported that chicken PGCs obtained from embryonic blood and gonads can be preserved in liquid nitrogen using freezing medium containing dimethyl sulfoxide (DMSO), and that these give rise to functional gametes via germline chimeras (Naito et al., 1994a; Tajima et al., 1998; Nakamura et al., 2010a). Recently, we have demonstrated that any of the commercially available DMSO-based cryoprotectants can be used to preserve chicken PGCs (Setioko et al., 2007). Additionally, viable offspring have originated from transferred PGCs which had been cryopreserved using the commercial DMSObased cryoprotectant, Cell Banker 1, as a freezing medium (Nakamura et al., 2010a). In this study, therefore, Cell Banker 1 was used in the freezing process. The average rate of recovered PGCs after freeze-thaw in the present study was greater than $54.3 \%$, comparable to the result of our previous study (Setioko et al., 2007). We suspected that some of the PGCs were broken by cryogenic damage and hence washed out during centrifugation. To recover the PGCs at a higher rate after freeze/thaw, it is important to reduce intracellular ice crystal formation and

Table 1. The number of recovered and viable primordial germ cells after freeze-thaw in each chicken breed

\begin{tabular}{|c|c|c|c|c|c|c|}
\hline \multirow{2}{*}{ Replication } & \multicolumn{2}{|c|}{ White Leghorn } & \multicolumn{2}{|c|}{ Barred Plymouth Rock } & \multicolumn{2}{|c|}{ Fayoumi } \\
\hline & Recovered & Viable & Recovered & Viable & Recovered & Viable \\
\hline 1 & 52 & 46 & 44 & 38 & 62 & 54 \\
\hline 2 & 43 & 36 & 45 & 40 & 50 & 43 \\
\hline 3 & 48 & 40 & 47 & 45 & 55 & 44 \\
\hline 4 & 46 & 38 & 42 & 36 & 62 & 54 \\
\hline 5 & 59 & 50 & 53 & 49 & 60 & 49 \\
\hline 6 & 61 & 52 & 55 & 46 & 56 & 48 \\
\hline 7 & 48 & 41 & 49 & 40 & 70 & 55 \\
\hline 8 & 53 & 42 & 56 & 44 & 67 & 57 \\
\hline 9 & 47 & 39 & 50 & 42 & 61 & 49 \\
\hline 10 & 47 & 40 & 47 & 41 & 61 & 51 \\
\hline 11 & 57 & 52 & 49 & 40 & 45 & 38 \\
\hline 12 & 40 & 34 & 58 & 49 & 54 & 45 \\
\hline 13 & 54 & 47 & 56 & 50 & 48 & 41 \\
\hline 14 & 57 & 50 & 48 & 43 & 51 & 46 \\
\hline 15 & 45 & 39 & 60 & 53 & 49 & 41 \\
\hline 16 & 44 & 40 & 53 & 48 & 63 & 55 \\
\hline 17 & 68 & 61 & 63 & 55 & 44 & 38 \\
\hline 18 & 65 & 55 & 67 & 56 & 60 & 50 \\
\hline 19 & 68 & 60 & 69 & 61 & 55 & 48 \\
\hline 20 & 65 & 54 & 61 & 54 & 46 & 40 \\
\hline $\mathrm{LSM} \pm \mathrm{SE}$ & $53.4 \pm 1.8$ & $45.8 \pm 1.6$ & $53.6 \pm 1.8$ & $46.5 \pm 1.6$ & $56.0 \pm 1.8$ & $47.3 \pm 1.6$ \\
\hline
\end{tabular}


Table 2. Percentage of viable primordial germ cells before and after freeze-thaw in each chicken breed

\begin{tabular}{|c|c|c|c|c|c|c|}
\hline \multirow{2}{*}{ Replication } & \multicolumn{2}{|c|}{ White Leghorn } & \multicolumn{2}{|c|}{ Barred Plymouth Rock } & \multicolumn{2}{|c|}{ Fayoumi } \\
\hline & Unfrozen $(\%)$ & Frozen $(\%)$ & Unfrozen $(\%)$ & Frozen $(\%)$ & Unfrozen $(\%)$ & Frozen $(\%)$ \\
\hline 1 & 97.0 & 88.5 & 100.0 & 86.4 & 97.0 & 87.1 \\
\hline 2 & 99.0 & 83.7 & 98.0 & 88.9 & 99.0 & 86.0 \\
\hline 3 & 98.0 & 83.3 & 100.0 & 95.7 & 98.0 & 80.0 \\
\hline 4 & 100.0 & 82.6 & 100.0 & 85.7 & 100.0 & 87.1 \\
\hline 5 & 99.0 & 84.7 & 96.0 & 92.5 & 99.0 & 81.7 \\
\hline 6 & 100.0 & 85.2 & 100.0 & 83.6 & 100.0 & 85.7 \\
\hline 7 & 99.0 & 85.4 & 100.0 & 81.6 & 99.0 & 78.6 \\
\hline 8 & 100.0 & 79.2 & 100.0 & 78.6 & 100.0 & 85.1 \\
\hline 9 & 100.0 & 83.0 & 99.0 & 84.0 & 100.0 & 80.3 \\
\hline 10 & 100.0 & 85.1 & 100.0 & 87.2 & 100.0 & 83.6 \\
\hline 11 & 100.0 & 91.2 & 100.0 & 81.6 & 100.0 & 84.4 \\
\hline 12 & 100.0 & 85.0 & 100.0 & 84.5 & 95.0 & 83.3 \\
\hline 13 & 99.0 & 87.0 & 99.0 & 89.3 & 97.0 & 85.4 \\
\hline 14 & 100.0 & 87.7 & 100.0 & 89.6 & 97.0 & 90.2 \\
\hline 15 & 100.0 & 86.7 & 100.0 & 88.3 & 100.0 & 83.7 \\
\hline 16 & 100.0 & 90.9 & 100.0 & 90.6 & 100.0 & 87.3 \\
\hline 17 & 100.0 & 89.7 & 100.0 & 87.3 & 99.0 & 86.4 \\
\hline 18 & 98.0 & 84.6 & 98.0 & 83.6 & 100.0 & 83.3 \\
\hline 19 & 98.0 & 88.2 & 98.0 & 88.4 & 96.0 & 87.3 \\
\hline 20 & 100.0 & 83.1 & 100.0 & 88.5 & 100.0 & 87.0 \\
\hline $\mathrm{LSM} \pm \mathrm{SE}$ & $99.4 \pm 0.6^{\mathrm{a}}$ & $85.7 \pm 0.6^{\mathrm{b}}$ & $99.4 \pm 0.6^{\mathrm{a}}$ & $86.8 \pm 0.6^{\mathrm{b}}$ & $98.8 \pm 0.6^{\mathrm{a}}$ & $84.7 \pm 0.6^{b}$ \\
\hline
\end{tabular}

Means with different superscript are significantly different $(P<0.05)$

Table 3. The number of primordial germ cells settled to the gonads of embryos at stage 27 befor and after freeze-thaw in each chicken breed

\begin{tabular}{|c|c|c|c|c|c|c|}
\hline \multirow{2}{*}{ Replication } & \multicolumn{2}{|c|}{ White Leghorn } & \multicolumn{2}{|c|}{ Barred Plymouth Rock } & \multicolumn{2}{|c|}{ Fayoumi } \\
\hline & Unfrozen & Frozen & Unfrozen & Frozen & Unfrozen & Frozen \\
\hline 1 & 41 & 19 & 25 & 15 & 35 & 13 \\
\hline 2 & 60 & 17 & 20 & 18 & 46 & 21 \\
\hline 3 & 42 & 25 & 19 & 9 & 46 & 21 \\
\hline 4 & 41 & 26 & 20 & 20 & 50 & 15 \\
\hline 5 & 38 & 25 & 37 & 10 & 31 & 16 \\
\hline 6 & 43 & 27 & 22 & 16 & 36 & 17 \\
\hline 7 & 55 & 28 & 24 & 15 & 34 & 15 \\
\hline 8 & 36 & 27 & 28 & 21 & 33 & 17 \\
\hline 9 & 50 & 24 & 23 & 13 & 41 & 17 \\
\hline 10 & 47 & 22 & 32 & 12 & 27 & 16 \\
\hline 11 & 43 & 30 & 40 & 14 & 37 & 24 \\
\hline 12 & 41 & 18 & 22 & 13 & 32 & 10 \\
\hline 13 & 50 & 24 & 31 & 11 & 30 & 23 \\
\hline 14 & 34 & 14 & 35 & 24 & 49 & 19 \\
\hline 15 & 38 & 26 & 17 & 17 & 24 & 20 \\
\hline 16 & 42 & 19 & 27 & 19 & 35 & 22 \\
\hline 17 & 45 & 20 & 26 & 12 & 43 & 14 \\
\hline 18 & 49 & 31 & 25 & 8 & 29 & 18 \\
\hline 19 & 31 & 27 & 31 & 9 & 44 & 25 \\
\hline 20 & 51 & 24 & 29 & 17 & 37 & 21 \\
\hline $\mathrm{LSM} \pm \mathrm{SE}$ & $43.9 \pm 1.3^{\mathrm{a}}$ & $23.7 \pm 1.3^{\mathrm{cd}}$ & $26.7 \pm 1.3^{\mathrm{c}}$ & $14.7 \pm 1.3^{\mathrm{ef}}$ & $37.0 \pm 1.3^{\mathrm{b}}$ & $18.2 \pm 1.3^{\mathrm{de}}$ \\
\hline
\end{tabular}

Means with different superscript are significantly different $(P<0.05)$ 

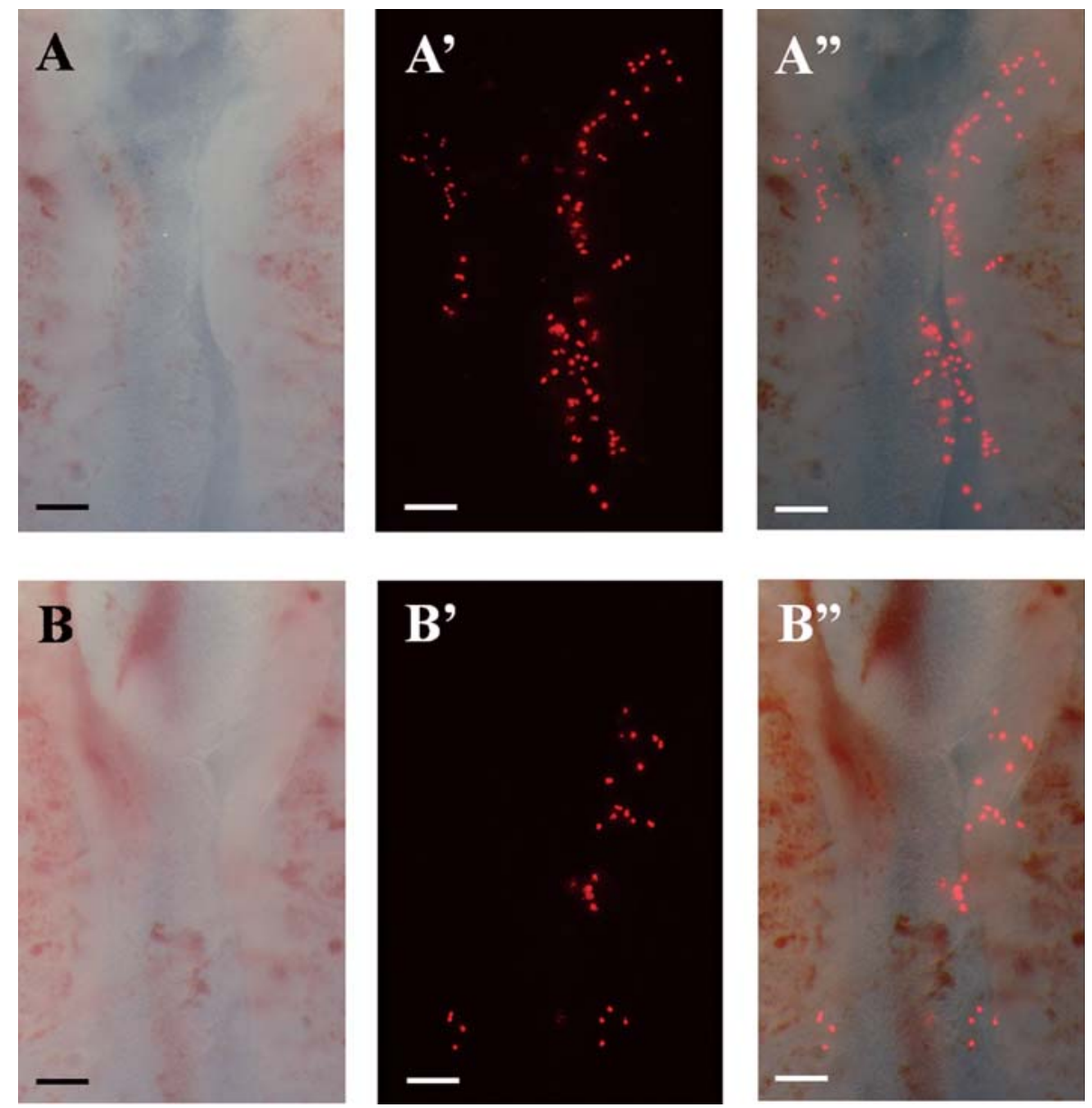

Fig. 1. Distribution of freshly collected and frozen-thawed primordial germ cells (PGCs) in the gonads of embryos at stage 27. Localization of transferred PGCs from White Leghorns in the embryonic gonads in unfrozen control group (A-A") and in frozen group $\left(B-B^{\prime \prime}\right)$. A and B: Bright field images of whole mounts of gonads. Gonadal migration of donor PGCs was tracked by staining with the fluorescent lipophilic carbocyanine dye PKH-26 ( $\left.\mathrm{A}^{\prime}, \mathrm{B}^{\prime}\right)$. $\mathrm{A}^{\prime \prime}, \mathrm{B}^{\prime \prime}$ : Merged image of $\mathrm{A}$ and $\mathrm{B}$, and $\mathrm{A}^{\prime}$ and $\mathrm{B}^{\prime}$. Bars $=100 \mu \mathrm{m}$.

cryogenic damage due to the high-concentration solutes that form when intracellular water freezes. This may be achieved using hydrophilic cryoprotectants to sequester water, and by freezing rapidly to minimize ice crystal growth (Freshney, 2005). In this study, sets of 100 PGCs were cryopreserved with $200 \mu \mathrm{l}$ freezing buffer to obtain accurate recovery and viability data after the freeze-thaw treatment. Viability of frozen-thawed PGCs has been reported to be as high as $94.2 \%$, considerably higher than that of our present study (Naito et al., 1994a). The report of them was conducted using much higher cell concentrations than those present in our study (about 5000 cells from early embryonic blood containing 3000 PGCs, cryopreserved in $40 \mu \mathrm{l}$ freezing buffer containing DMSO). This difference in cell concentration is sufficient to explain the difference in viability between our current study and them.

Our PGC migration assays indicate that PGCs cryopreserved in commercial cryoprotectant are biologically functional. The number of PGCs settled in the gonads of embryos at stage 27 decreased to $52.8 \%$ (relative to the control group) after freezing and thawing treatment. Even allowing for the difference in viability of transferred PGCs between the frozen and control groups, frozenthawed PGCs exhibit a decline in their functionality. Freeze-thaw treatment is considered to lower the migration capability and/or proliferating potential of PGCs. This should lead to a depression of germline transmission efficiency in chimeric chickens. Recently, we have developed an efficient method for delivering busulfan (1,4- 
butanediol dimethanesulfonate), an alkylating agent with cytotoxic effect on chicken germ cells, to the early chicken embryo (Nakamura et al., 2008). This method allows removal of the endogenous PGCs and repopulation with exogenous PGCs in chicken embryos at early developmental stages (Nakamura et al., 2009). Subsequently, we have exploited these advances for production of chimeric chickens in which recipient germlines were replaced by donor cells with $99.5 \%$ transfer success (Nakamura et al., 2010b). Meanwhile, the germline transmission rate is reportedly improved by increasing the number of donor PGCs transferred into recipient embryos (Naito et al., 1999). Therefore, removing endogenous PGCs of recipient embryos and/or increasing the number of transferring PGCs appears to enhance the efficiency of germline transmission of chimeras generated from frozen-thawed PGCs.

In this study, three chicken breeds, WL, BPR and FA, were used as conservation models. No significant difference between the three breeds was observed in the recovery and viability rates of PGCs following freezing and thawing. Interestingly, in the control group only, the number of settled PGCs in the gonads of embryos after 5 days incubation varied significantly between the breeds. The average numbers of PGCs settled in stage 27 gonads were highest for the WL breed, and lowest for the BPR breed. Although the same trend was observed in the frozen group, the differences were below the level of significance, presumably because overall numbers of PGCs used for statistical analysis were low in this group. These results strongly indicate that transmute ability of PGCs to migrate into the gonads and the proliferative ability of PGCs after settlement in the gonads are different between breeds. Our most recent study showed that the efficiency of generating viable offspring derived from donor PGCs is markedly enhanced when embryos with a low number of endogenous PGCs are used as recipients (Nakamura et al., 2010a). On the other hand, Naito et al. (1994b) reported a more than 3.5 times increase in frequency of viable donor-derived progenies after transfer of WL PGCs into BPR recipients than after transfer of vice versa, though the number of endogenous PGCs at the time of donor PGCs transfer was about 1.7 times higher in BPR than in WL (Naito et al., 1994b). These authors suggested that removal of blood containing PGCs from recipients prior to transfer of donor PGCs might be more effective when the number of endogenous PGCs is high. Therefore, the results and conclusions of Naito et al. are consistent with those of our own studies.

Viability tests of recovered PGCs revealed that $46.5 \%$ of the total PGCs which had been frozen by the method of slow-freezing were available after thawing. Frozenthawed PGCs are biologically functional enough to migrate and settle in the gonads after transfer, however, migration capability and/or proliferation capacity of these cells are reduced by cryogenic damage. Furthermore, PGC functionality differs between chicken breeds. Further studies on cryoprotectant use and optimization of freezing condition are required to improve the efficiency of PGC transfer after freeze-thaw treatment.

\section{Acknowledgments}

This work was funded by a Research Fellowship for Young Scientists from the Japan Society for the Promotion of Sciences. The authors wish to thank the staff of the Poultry Management Section of the NILGS for bird maintenance and for providing the fertilized eggs. We are grateful to Dr. K. Hamano of the Faculty of Agriculture, Shinshu University, for his helpful advice. The present study was supported by all members of the Animal Breeding and Reproduction Research Team, NILGS and all colleagues at the Laboratory of Animal Developmental Genetics, Faculty of Agriculture, Shinshu University.

\section{References}

Eyal-Giladi $\mathrm{H}$ and Kochav S. From cleavage to primitive streak formation: a complementary normal table and a new look at the first stages of the development of the chick. I. General morphology. Developmental Biology, 49: 321-337. 1976.

Freshney RI. Culture of Animal Cells. $2^{\text {nd }}$ ed. pp.245-256. Alan R Liss, New York. 1987.

Fujimoto T, Ukeshima A and Kiyofuji R. The origin, migration and morphology of primordial germ cells in the chicken embryo. Anatomical Record, 185: 139-145. 1976.

Hamburger V and Hamilton HL. A series of normal stages in the development of the chick embryo. Journal of Morphology, 88: 49-92. 1951.

Hammerstedt RH and Graham JK. Cryopreservation of poultry sperm: the enigma of glycerol. Cryobiology, 29: 26-38. 1992.

Kagami H, Tagami T, Matsubara Y, Harumi T, Hanada H, Maruyama K, Sakurai M, Kuwana T and Naito M. The developmental origin of primordial germ cells and the transmission of the donor-derived gametes in mixed-sex germline chimeras to the offspring in the chicken. Molecular Reproduction and Development, 48: 501-510. 1997.

Kuwana T, Hashimoto K, Nakanishi A, Yasuda Y, Tajima A and Naito M. Long-term culture of avian embryonic cells in vitro. International Journal of Developmental Biology, 40: 1061-1064. 1996.

Kuwana T, Kawashima T, Naito M, Yamashita H, Matsuzaki M and Takano T. Conservation of a threatened indigenous fowl (Kureko Dori) using the germline chimeras transplanted from primordial germ cells. Journal of Poultry Science, 43: 60-66. 2006.

Lake PE and Stewart JM. Preservation of fowl semen in liquid nitrogen - an improved method. British Poultry Science, 19: 187-194. 1978.

Mayanagi T, Kurosawa R, Ohnuma K, Ueyama A, Ito K and Takahashi J. Purification of mouse primordial germ cells by Nycodenz. Reproduction, 125: 667-675. 2003.

Mozdziak PE, Angerman-Stewart J, Rushton B, Pardue SL and Petitte JN. Isolation of chicken primordial germ cells using fluorescence-activated cell sorting. Poultry Science, 84: 594600. 2005.

Naito M, Matsubara Y, Harumi T, Tagami T, Kagami H, Sakurai M and Kuwana T. Differentiation of donor primordial germ cells into functional gametes in the gonads of 
mixed-sex germline chimaeric chickens produced by transfer of primordial germ cells isolated from embryonic blood. Journal of Reproduction and Fertility, 117: 291-298. 1999.

Naito M, Tajima A, Tagami T, Yasuda Y and Kuwana T. Preservation of chick primordial germ cells in liquid nitrogen and subsequent production of viable offspring. Journal of Reproduction and Fertility, 102: 321-325. 1994a.

Naito M, Tajima A, Yasuda Y and Kuwana T. Production of germline chimeric chickens, with high transmission rate of donor-derived gametes, produced by transfer of primordial germ cells. Molecular Reproduction and Development, 39: 153-161. 1994b.

Nakamura Y, Usui F, Atsumi Y, Otomo A, Teshima A, Ono T, Takeda K, Nirasawa K, Kagami H and Tagami T. Effects of busulfan sustained-release emulsion on depletion and repopulation of primordial germ Cells in early chicken embryos. Journal of Poultry Science, 46: 127-135. 2009.

Nakamura Y, Usui F, Miyahara D, Mori T, Ono T, Takeda K, Nirasawa K, Kagami $\mathrm{H}$ and Tagami T. Efficient system for preservation and regeneration of genetic resources in chicken: concurrent storage of primordial germ cells and live animals from early embryos of a rare indigenous fowl (Gifujidori). Reproduction, Fertility and Development, 22: 1237-1246. 2010a.

Nakamura Y, Usui F, Ono T, Takeda K, Nirasawa K, Kagami H and Tagami T. Germline replacement by transfer of primordial germ cells into partially sterilized embryos in the chicken. Biology of Reproduction, 83: 130-137. 2010b.

Nakamura Y, Yamamoto Y, Usui F, Atsumi Y, Ito Y, Ono T, Takeda $\mathrm{K}$, Nirasawa $\mathrm{K}$, Kagami $\mathrm{H}$ and Tagami $\mathrm{T}$. Increased proportion of donor primordial germ cells in chimeric gonads by sterilisation of recipient embryos using busulfan sustained-release emulsion in chicken. Reproduction, Fertility and Development, 20: 900-907. 2008.

Nakamura Y, Yamamoto Y, Usui F, Mushika T, Ono T, Setioko AR, Takeda K, Nirasawa K, Kagami $\mathrm{H}$ and Tagami T. Migration and proliferation of primordial germ cells in the early chicken embryo. Poultry Science, 86: 2182-2193. 2007.

Ono T and Machida Y. Immunomagnetic purification of viable primordial germ cells of Japanese quail (Coturnix japonica). Comparative Biochemistry and Physiology (Part A), 122: 255-259. 1999.
Park TS, Jeong DK, Kim JN, Song KH, Hong YH, Lim JM and Han JY. Improved germline transmission in chicken chimeras produced by transplantation of gonadal primordial germ cells into recipient embryos. Biology of Reproduction, 68: 1657-1662. 2003.

Petitte JN, Clark ME, Liu G, Verrinder Gibbins AM and Etches RJ. Production of somatic and germline chimeras in the chicken by transfer of early blastodermal cells. Development, 108: 185-189. 1990.

Setioko AR, Tagami T, Tase H, Nakamura Y, Takeda K, and Nirasawa K. Cryopreservation of primordial germ cells (PGCs) from White Leghorn embryos using commercial cryoprotectants. Journal of Poultry Science, 44: 73-77. 2007.

Tajima A, Naito M, Yasuda Y and Kuwana T. Production of germ line chimera by transfer of primordial germ cells in the domestic chicken (Gallus domesticus). Theriogenology, 40: 509-519. 1993.

Tajima A, Naito M, Yasuda Y and Kuwana T. Production of germ-line chimeras by transfer of cryopreserved gonadal primordial germ cells (gPGCs) in chicken. Journal of Experimental Zoology, 280: 265-267. 1998.

Tsunekawa N, Naito M, Sakai Y, Nishida T and Noce T. Isolation of chicken vasa homolog gene and tracing the origin of primordial germ cells. Development, 127: 27412750. 2000.

Vick L, Luke G and Simkiss K. Germ-line chimaeras can produce both strains of fowl with high efficiency after partial sterilization. Journal of Reproduction and Fertility, 98: 637 $-641.1993$.

Yamamoto Y, Usui F, Nakamura Y, Ito Y, Tagami T, Nirasawa K, Matsubara Y, Ono T and Kagami H. A novel method to isolate primordial germ cells and its use for the generation of germline chimeras in chicken. Biology of Reproduction, 77: 115-119. 2007.

Yasuda Y, Tajima A, Fujimoto T and Kuwana T. A method to obtain avian germ-line chimeras using isolated primordial germ cells. Journal of Reproduction and Fertility, 96: 521528. 1992.

Zhao DF and Kuwana T. Purification of avian circulating primordial germ cells by nycodenz density gradient centrifugation. British Poultry Science, 44: 30-35. 2003. 